\title{
Early handling attenuates enhancement of glucocorticoid receptors in the prefrontal cortex in an animal model of post-traumatic stress disorder
}

Sophie A George ${ }^{1 *}$, Stephanie A Stout ${ }^{1}$, Melissa Tan ${ }^{1}$, Dayan Knox ${ }^{1,3}$ and Israel Liberzon ${ }^{1,2}$

\begin{abstract}
Background: Changes in glucocorticoid receptors (GRs) have been implicated in the pathogenesis of stress related psychiatric disorders such as depression and post-traumatic stress disorder (PTSD). Abnormal adaptation of the stress-response system following traumatic stress can lead to an altered hypothalamic-pituitary-adrenal axis that may contribute to PTSD development. Indeed, elevated GR expression in the hippocampus and prefrontal cortex linked to PTSD-like characteristics have been reported in the validated animal model of PTSD, single-prolonged stress. These findings implicate increased levels of GRs in the development of post-traumatic psychopathology and suggest that exploration of GR-targeted interventions may have potential for PTSD prevention. Early handling during the neonatal phase alters GR expression and is proposed to confer resilience to stress. We therefore examined the effects of combined early handling and single prolonged stress treatments on GR expression.
\end{abstract}

Methods: Timed pregnant dams gave birth to pups that were subjected to early handling $(n=11)$ or control $(n=13)$ procedures during the neonatal phase. At postnatal day 45 animals underwent single prolonged stress or a control procedure. Rats were euthanized one day later and GR levels were assayed using western blot electrophoresis.

Results: Single prolonged stress exposure enhanced GR expression in the hippocampus and prefrontal cortex. Early handling treatment protected against single prolonged stress-induced enhancement of GR expression in the prefrontal cortex, but not in the hippocampus.

Conclusions: These data are a first step in highlighting the importance of targeting GR systems in prevention/ resilience and may suggest that preventive strategies targeting GR upregulation might be particularly effective when prefrontal rather than hippocampal GRs are the target.

Keywords: Hippocampus, HPA axis, Maternal care, Single prolonged stress, Stress

\section{Background}

Stress initiates a cascade of neuroendocrine events in the hypothalamic-pituitary-adrenal (HPA) axis, which ultimately leads to increased secretion of the glucocorticoid hormone cortisol from the adrenal glands. Activity of the HPA axis is tightly controlled through complex regulatory mechanisms of glucocorticoid negative

\footnotetext{
* Correspondence: alicegeo@med.umich.edu

'Department of Psychiatry, University of Michigan, 4250 Plymouth Road, Ann Arbor, Ml 48109, USA

Full list of author information is available at the end of the article
}

feedback. Glucocorticoids regulate the secretion of corticotropin-releasing factor and adrenocorticotropic hormone, from the hypothalamus and pituitary, respectively [1-4]. In addition, receptor sites within the hippocampus and prefrontal cortex play an important role in the regulation of HPA axis activity [2,5]. Following chronic or traumatic stress, inappropriate adaptation of the HPA axis can lead to pathological states; specifically, changes in glucocorticoid receptors (GRs) have been implicated in the pathogenesis of stress related psychiatric disorders such as post-traumatic stress disorder (PTSD) [6] and symptoms

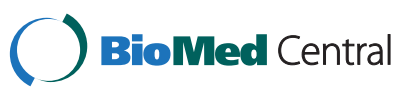


of PTSD are believed to reflect trauma-induced changes that lead to long-term dysfunctional stress regulation [7-9].

PTSD is characterized by increased cortisol suppression to dexamethasone, believed to result from an increased number or sensitivity of GRs [10]. Recently, in a prospective study, van Zuiden et al. reported higher levels of GR as a risk factor for subsequent development of PTSD in a sample of soldiers [11,12]. Findings from animal models further support changes in GR as the potential mechanism for the development of PTSD symptoms. In addition to reproducing cardinal symptoms of PTSD, such as hyperarousal and elevated fast feedback of the HPA axis [13-16], increased GR levels have been found in the single prolonged stress (SPS) [16-18] and predator exposure models in the hippocampus and prefrontal cortex [19]. In concert, pretreatment with GR antagonists prevents PTSD-like phenotypes in both SPS and predator exposure models $[14,20]$. Furthermore, in a recent "dismantling" study in which full SPS (involving restraint, forced swim, and ether exposure) was compared to the effect of different components of SPS (i.e., two of three stressors), only those animals that were exposed to the full SPS procedure and demonstrated the greatest degree of upregulation of GR in the hippocampus and prefrontal cortex, exhibited deficits in retention of extinction memories - a mechanism that is proposed to contribute to an inability to retain new safe memories and prevent recovery from trauma [19,21,22]. Together, these findings implicate altered GRs in the development of some aspects of posttraumatic psychopathology, and suggest that exploration of GR-targeted interventions may have potential for PTSD resilience/prevention.

Levine [23-25], and subsequently others (e.g., [26]), demonstrated that glucocorticoid responses to stress were modulated by early life environmental events and could result in stable changes to HPA axis reactivity, most notably via alterations in GR gene expression in the hippocampus and frontal cortex [27]. Early handling (EH), which involves brief daily separation from the mother during the neonatal phase is one such manipulation that has a documented effect on GR expression. EH increases the frequency of maternal behaviors $[28,29]$ and thus increases GR expression and confers resilience to later stress [30,31]. Meany et al. demonstrated that EH enhances the availability of GRs [32], which in turn attenuates stressinduced HPA axis responsivity, as evidenced by attenuated glucocorticoid release in response to stress and reduced anxiety-like behaviors in adulthood [23,27,30,32].

While a number of previous studies have demonstrated that EH can attenuate the effects of chronic stress on inducing HPA axis reactivity [33-35], the effects of EH in animal models of PTSD have not been examined. Given the documented role of GR upregulation in the etiology of PTSD and the demonstration that "traumatic" stress as described in the SPS model increases GR expression, we hypothesized that EH would protect against the GR enhancement that develops following SPS. The goal of this study was to examine the combined effects of $\mathrm{EH}$ and single prolonged stress on GR expression. We chose to examine GR changes in the hippocampus and prefrontal cortex because of their documented role in the protective effect of EH [27], as well in the development of SPSinduced changes following traumatic stress $[16,19]$.

\section{Methods}

\section{Animals}

Timed-pregnant dams (Charles River, Portage, MI, USA) were delivered to the Veterans Affairs Veterinary Medical Unit at approximately gestation day 16 . Dams were singly housed in a temperature and humidity controlled environment, on a 12 hour light-dark cycle, and had ad lib access to standard laboratory chow and water. All experimental procedures were approved by the Veteran Affairs Institutional Animal Care Usage Committee and were in accordance with the National Institute of Health Guide for the Care and Use of Laboratory Animals. The day of birth of the litter was marked as postnatal day (PND) 0 . Litter sizes varied naturally between 6 and 12, and on PND 2, animals were culled to ensure that equivalent numbers of males and females were present in each litter. The animals in this experiment were drawn from eight litters, and the number of animals in each litter from which data was sampled ranged from 4-12. Pups were subjected to EH or animal facility reared (AFR) treatments [36]. Briefly, EH litters received 15 minutes of daily maternal separation for 21 days. AFR rats were left undisturbed, except for biweekly cage maintenance. On PND 23, pups were weaned and housed in same sex sibling pairs.

\section{SPS and brain homogenate preparation}

On PND 45, 24 male Sprague-Dawley rats were assigned to the SPS (AFR = 7, EH = 5) or control $(\mathrm{AFR}=6, \mathrm{EH}=6)$ groups. SPS rats were exposed to two hours of restraint, followed by 20 minutes of forced swimming in a $55 \mathrm{~L}$ container. After 15 minutes recuperation rats were exposed to $70 \mathrm{~mL}$ of ether in a desiccator until general anesthesia was induced (typically less than five minutes). Rats were then returned to their home cages for a seven day quiescent period. The SPS procedure refers to the application of the three stressors plus the seven day quiescent period. The quiescent period has been demonstrated to be critical for the development of PTSD-like physiological and behavioral abnormalities following SPS [15,37]. Animals assigned to the control group were left undisturbed in their home cages for the duration of SPS.

Following SPS (i.e., 8 days after the application of acute stressors), rats were euthanized by rapid decapitation, their brains were removed, flash frozen in chilled 
isopentane and stored in a $-80^{\circ} \mathrm{C}$ freezer for later processing. Brains were then thawed to $-20^{\circ} \mathrm{C}$ in a cryostat and the prefrontal cortex was dissected, approximately $1.00 \mathrm{~mm}$ anterior to Bregma [38]. The cerebrum was separated from the brain stem, thawed on ice, and the hippocampus was removed. The prefrontal cortex and hippocampus were sonicated separately in homogenization buffer (50 mM Trizma base, $1 \mathrm{mM}$ ethylenediaminetetraacetic acid, $10 \%$ sucrose, $4 \%$ sodium dodecyl sulfate, $2 \mathrm{X}$ protease inhibitor cocktail (Roche USA), pH 7.0 to 7.4), centrifuged at 105,000 $\mathrm{x} g$ for 45 minutes, homogenates decanted, and protein content determined using a Pierce BCA kit (Sigma-Aldrich, St. Louis, MO, USA). Approximately $40 \mu \mathrm{g}$ of protein was diluted into a $1 \mathrm{X}$ Lamelli sample buffer and stored in a $-80^{\circ} \mathrm{C}$ freezer until the western blot assay was performed.

\section{Western blot electrophoresis}

Western blot for total GR (cytoplasm and nucleus) was adapted from Spencer et al. [39] and conducted as previously described [19]. Briefly, samples heated at $70^{\circ} \mathrm{C}$ for 7 minutes were electrophoresed on $7.5 \%$ Tris $\mathrm{HCl}$ gels (Bio-Rad Laboratories, Inc., Hercules, CA, USA) along with a molecular weight ladder (Li-COR, Lincoln, NE, USA). Proteins in gels were transferred onto nitrocellulose membranes and blocked in blocking buffer (BB) (5\% non-fat milk and $0.05 \%$ Tween-20 in trisbuffered saline (TBS)). Nitrocellulose membranes were then probed for GR by incubating membranes with a rabbit polyclonal GR antibody (Santa Cruz Biotechnology Inc., Santa Cruz, CA, USA; M-20, diluted 1:500 in $\mathrm{BB}$ ) for 2 hours. After several washes in $0.05 \%$ Tween-20 in TBS, nitrocellulose membranes were incubated with an IRDye 800 conjugated anti-rabbit IgG secondary antibody ( $\mathrm{Li}-\mathrm{COR}$, diluted 1:2,000 in BB) for 1 hour. Nitrocellulose membranes were then rinsed with TBS and scanned using a Li-COR Odyssey Scanner for visualization of GR bands.

After probing nitrocellulose membranes for GR, the same membranes were probed for actin related protein
(Arp) which was used as the reference protein as previously described [40]. Nitrocellulose membranes were incubated with a rabbit polyclonal Arp antibody (Santa Cruz Antibodies, Arp-2, diluted 1:2,000 in BB), washed in 0.05\% Tween-20 in TBS, and then incubated with the secondary antibody (Li-COR, 1:8,000 in BB). Nitrocellulose membranes were rinsed with TBS and scanned in a Li-COR Odyssey scanner in order to visualize Arp bands.

Images of scanned nitrocellulose membranes were analyzed using Odyssey software (Li-COR). The integrated intensity of the GR and Arp bands were expressed as a ratio (GR/Arp) and used as a relative measure of GR levels. Each gel contained representative samples from each of the treatment groups (Additional file 1). Samples were initially run in duplicate, but after a small coefficient of variance was established, single samples were run subsequently. GR levels were subjected to two factor analysis with the factors neonatal treatment (EH vs. AFR) and stress treatment (SPS vs. control). GR in the hippocampus and prefrontal cortex were analyzed separately. Main and simple effects were analyzed using analysis of variance (ANOVA), while main and simple comparisons were analyzed using $t$-test with a Bonferroni correction where necessary. Criterion of significance for all tests was set at $P<0.05$.

\section{Results}

Prominent bands were observed between the $100 \mathrm{kDa}$ and $75 \mathrm{kDa}$ molecular weight markers for GR, and $50 \mathrm{kDa}$ and $37 \mathrm{kDa}$ for Arp in both hippocampus and prefrontal cortex (Figure 1). These bands correspond closely to previously determined locations for GR and Arp using the primary antibodies described in the Methods section.

An ANOVA of GR expression in the prefrontal cortex revealed a significant SPS $\times \mathrm{EH}$ interaction $\left(\mathrm{F}_{(1,20)}=7.077\right.$, $P=0.015)$. Post hoc comparisons revealed higher GR signal in SPS animals in comparison to controls in AFR treated groups $\left(t_{(11)}=2.856, P=0.016\right)$, but this effect was not present in the EH exposed groups $\left(\mathrm{t}_{(9)}=0.626\right.$, $P=0.547)$, suggesting that SPS-induced enhancement of

\section{AFR}

Hippocampus Prefrontal Cortex

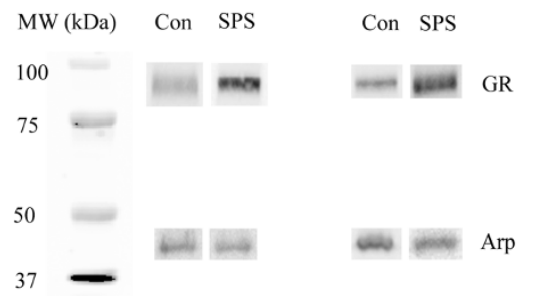

EH

Hippocampus Prefrontal Cortex

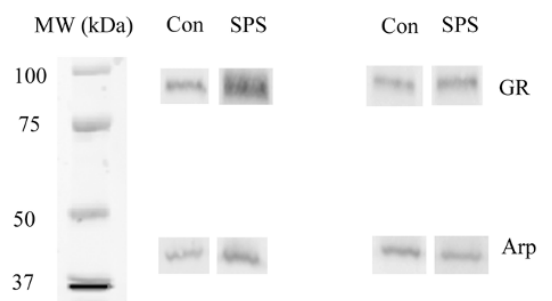

Figure 1 Representative protein bands from all treatment groups in this study. MW: Molecular weight markers; AFR: Animal facility reared; EH: Early handling; SPS: Single prolonged stress; Con: Control; GR: Glucocorticoid receptor; Arp: Actin related protein. 
GR expression in prefrontal cortex was effectively prevented by EH. An ANOVA of hippocampal GR revealed a significant main effect of SPS $\left(\mathrm{F}_{(1,17)}=4.929, P=0.04\right)$ with higher GR signal in SPS-exposed animals; however, there was no SPS $\times \mathrm{EH}$ interaction $\left(\mathrm{F}_{(1,17)}=1.487\right.$, $P=0.239)$ or main effect of $\mathrm{EH}\left(\mathrm{F}_{(1,17)}=0.851, P=0.369\right)$, suggesting that EH did not attenuate SPS-induced increases in GR expression in the hippocampus. These data are illustrated in Figure 2.

\section{Discussion}

In the present experiments SPS enhanced GR expression in the hippocampus and prefrontal cortex, replicating findings previously reported by ourselves and others $[16,18,19]$. EH, on the other hand, attenuated SPS-induced enhancement of GR in the prefrontal cortex, suggesting that EH may be protective against some of the SPSinduced changes implicated in PTSD pathophysiology. Interestingly, while affecting GR in prefrontal cortex, EH did not attenuate SPS-induced enhancement of GR in the hippocampus suggesting that there are regional differences in GR expression following combined effects of early life environment and stressors experienced in adulthood. It has long been suggested that early life experiences may lead to developmental changes that result in stable alterations to HPA axis and potentially confer resilience to later stress. To our knowledge, this is the first report of the combined effects of early life experiences and later "traumatic" stress on GR expression. Given the established role of GR in HPA axis regulation and stress reactivity $[30,31]$ as well as in the pathophysiology of the SPS model of PTSD [16], these findings may have some implications for understanding mechanisms of resilience to traumatic stress, and of the regional differences that may be critical in moderating the protective effect of early life experiences to later life stressors. While intriguing, the functional implications of these GR expression changes will need to be examined in order to further establish the significance of

\section{Hippocampus}

\section{Prefrontal Cortex}

A

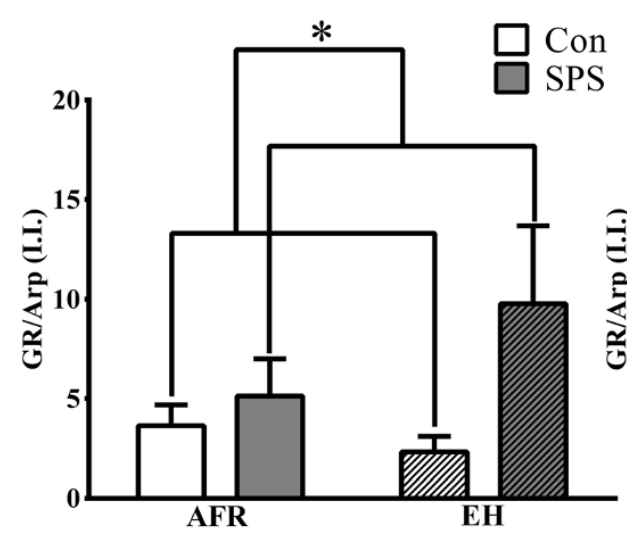

B

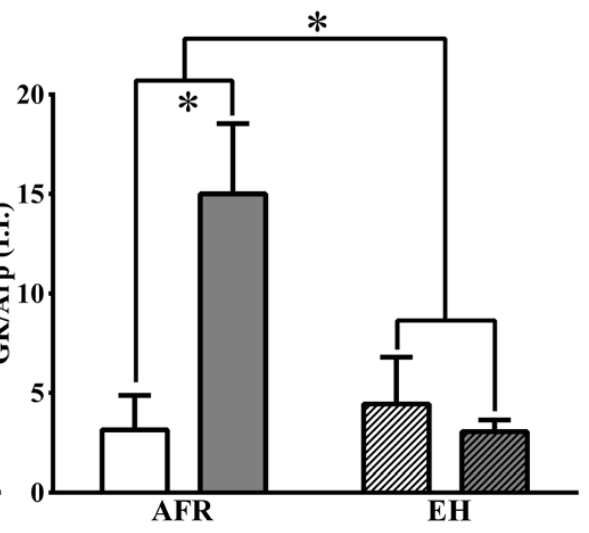

D
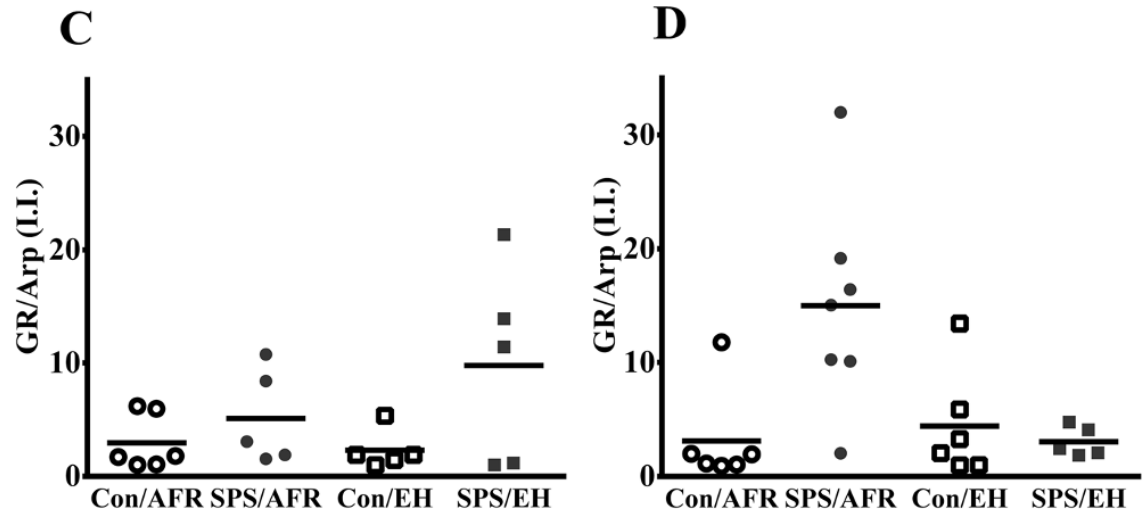

Figure 2 The effects of early handling (EH) and single prolonged stress on mean relative glucocorticoid levels in the (A) hippocampus and (B) prefrontal cortex. (C,D) Scatter plots showing individual data points from animals included in this experiment. *P $<0.05$. AFR: Animal facility reared; EH: Early handling; SPS: Single prolonged stress; Con: Control; GR: Glucocorticoid receptor; Arp: Actin related protein; I.I.: Integrated intensity. 
this finding. In addition, given the heterogeneity of the prefrontal cortex, in particular with respect to regulation of stress reactivity conferred by environmental influences [41], it would be interesting to further examine whether different subregions of the prefrontal cortex contribute differentially to the effect reported herein.

Differential changes in GR expression between frontal cortex and hippocampus following stress manipulations have been previously reported by our laboratory [19] as well as by others. Indeed, in Meaney's seminal paper in which the effect of EH on GR in the frontal cortex and hippocampus is first described, GR in the hippocampus was increased in EH animals compared to non-handled controls irrespective of post-weaning housing conditions [32]. However, this was not the case in the frontal cortex in which post-weaning housing condition moderated GR expression. These data suggest that hippocampal changes in GR may be more stable and enduring than those in the frontal cortex, resonating with our own finding, in which hippocampal GRs were found to be less sensitive to environmental effects than GRs in the prefrontal cortex.

The precise functional role of hippocampal and prefrontal cortex GRs are not known, although a wealth of data suggests that receptor sites within the hippocampus and prefrontal cortex play an important role in the regulation of HPA axis activity [2,5]. Recent data from our own laboratory, in which full SPS (comprised of all three stressors) was compared to partial SPS procedures (e.g., restraint + ether or forced swim + restraint), demonstrated that exposure to ether alone was sufficient to alter prefrontal GR levels, while multiple combined stressors were required to alter GR levels in the hippocampus. Moreover, the behavioral data from this study indicated that the combined effect of serial exposure to all three stressors (restraint, forced swim and ether) was required in order to observe extinction retention deficits. These results suggest that the mere enhancements in GR expression in the hippocampus and prefrontal cortex might be insufficient to lead to PTSD-relevant behavioral deficits, but "threshold" change in these regions is required for SPS-induced extinction retention deficits to manifest. Together with the present data, these findings suggest that the ability of EH to attenuate SPS-induced enhancement in prefrontal GR levels should be interpreted with caution as they may not necessarily translate to resilience in PTSD-relevant behavioral outcomes. Addressing this question directly, for instance by examining the effect of $\mathrm{EH}$ on extinction retention deficits in SPS animals, will be an important goal of future studies.

Interestingly, in these experiments we did not detect effects of EH alone on total GR expression. This is in contrast to the findings reported by Meaney et al., in which EH was found to increase baseline levels of unbound cytoplasmic GR. There are several possibilities that may explain this apparent discrepancy; EH effects are known to be mediated by FKBP5 protein modulating GR sensitivity to ligands [42]. Thus, when FKBP5 is bound to GR, binding of glucocorticoids to GRs is reduced. It is therefore possible that EH could increase GR sensitivity by attenuating FKBP5-GR binding. Because radioimmunoassays are typically used to assay unbound cytoplasmic GR, these assays rely on protein-ligand binding and therefore a treatment that increases GR sensitivity could be interpreted as an increase in unbound cytoplasmic GR. Thus, the differing approaches to measuring GR levels may explain these apparently contradictory findings. Alternatively, there were a number of other methodological differences that may underlie the difference in baseline EH findings between the two studies. For example, different strains of rat were used and the age at which GR was measured was different, as were post-weaning housing conditions, all of which have been suggested to impact GR expression [32].

Interestingly, in Meaney's model [32], increases in GR expression are interpreted as functionally beneficial, with $\mathrm{EH}$ increasing GR expression and conferring later resilience to stress. Accordingly, prolonged maternal separation, which reduces GR expression, is proposed to have adverse consequences, resulting in vulnerability to later stress. Conversely, our data suggest that GR increases following SPS relate to greater functional impairment [19]. The differences in the developmental stages at which GR changes are initiated may be critical to the behavioral impact of GR changes, explaining the seemingly conflicting results. The present data shows that $\mathrm{EH}$ prevents traumainduced increases in GR in adult fully-grown animals, thus suggesting that early life EH protects against later increases in GR, possibly because of a more efficient negative feedback system which clamps down the HPA axis response following traumatic stress. Critically, both studies confirm EH results in changes in GR expression that likely result in resilience but further research is clearly needed to examine the precise mechanisms by which $\mathrm{EH}$ modulates GR expression following different stressors and in different brain regions.

\section{Conclusions}

While a number of previous studies have demonstrated that EH can attenuate the effects of chronic stress on inducing HPA axis reactivity, to our knowledge, this is the first study to examine the effects of $\mathrm{EH}$ in an animal model of PTSD. The data reported here suggest that the early life environment may have an important role in later responses to traumatic stress, and suggest that regional differentiation in GR expression may be important characteristic of the effects. These data, while limited to a measure of protein expression, underscore the importance of targeting GR systems in prevention/resilience and suggest 
that preventive strategies targeting GR upregulation may be more effective when prefrontal rather than hippocampal GRs are the target.

\section{Additional file}

Additional file 1: Representative example of a western blot assay. The data presented in this manuscript were obtained from a larger research project that examined the effects of a number of neonatal treatments and single prolonged stress on GR expression in the hippocampus and prefrontal cortex. This additional data file shows a representative example of a western blot assay.

\section{Abbreviations}

AFR: Animal facility reared; Arp: Actin related protein; BB: Blocking buffer; EH: Early handling; GR: Glucocorticoid receptor; HPA: Hypothalamic-pituitary-adrenal axis; PND: Postnatal day; PTSD: Post-traumatic stress disorder; SPS: Single prolonged stress; TBS: Tris-buffered saline.

\section{Competing interests}

The authors declare that they have no competing interests.

\section{Author contributions}

SG was the primary writer of the manuscript. She also contributed to data collection, statistical analyses and interpretation of the data. DK contributed to the conception and design of the experiment, statistical analyses and interpretation of the data. He supervised the data acquisition and substantially contributed to the drafting and revision of the manuscript. SS and MT were primarily responsible for acquisition of data. They conducted all early handling protocols and stress procedures and carried out initial statistical analyses. IL bore overall responsibility for conception and design of the study, and interpretation of the data. He made critical revisions to the manuscript. All authors read and approved the final manuscript.

\section{Acknowledgements}

The research in this manuscript was supported by a VA Merit award and Department of Defense grant W81XWH-08-1-0661 awarded to Israel Liberzon. We would like to thank Dr. Sarah Clinton for her valuable contribution.

\section{Author details}

'Department of Psychiatry, University of Michigan, 4250 Plymouth Road, Ann Arbor, MI 48109, USA. ${ }^{2}$ Ann Arbor Veterans Affairs Hospital, 2215 Fuller Road, Ann Arbor, MI 49105, USA. ${ }^{3}$ Present address: Department of Psychology, University of Delaware, 108 Wolf Hall, Newark, DE 19716, USA.

\section{Received: 17 July 2013 Accepted: 29 October 2013}

Published: 2 December 2013

\section{References}

1. Dallman MF, Akana SF, Jacobson L, Levin N, Cascio CS, Shinsako J: Characterization of corticosterone feedback regulation of $\mathrm{ACTH}$ secretion. Ann N Y Acad Sci 1987, 512:402-414.

2. de Kloet ER: Brain corticosteroid receptor balance and homeostatic control. Front Neuroendocrinol 1991, 12:95-164.

3. Jacobson L, Sapolsky R: The role of the hippocampus in feedback regulation of the hypothalamic-pituitary-adrenocortical axis. Endocr Rev 1991, 12:118-134.

4. Feldman S, Weidenfeld J: Neural mechanisms involved in the corticosteroid feedback effects on the hypothalamo-pituitary-adrenocortical axis. Prog Neurobiol 1995, 45:129-141.

5. Diorio D, Viau V, Meaney MJ: The role of the medial prefrontal cortex (cingulate gyrus) in the regulation of hypothalamic-pituitary-adrenal responses to stress. J Neurosci 1993, 13:3839-3847.

6. Yehuda R: Biology of posttraumatic stress disorder. J Clin Psychiatry 2001, 62(Suppl 17):41-46.

7. Heim C, Nemeroff CB: Neurobiology of posttraumatic stress disorder. CNS Spectr 2009, 14:13-24.

8. Yehuda R: Clinical relevance of biologic findings in PTSD. Psychiatr Q 2002, 73:123-133.
9. de Kloet CS, Vermetten E, Geuze E, Kavelaars A, Heijnen CJ, Westenberg HG: Assessment of HPA-axis function in posttraumatic stress disorder: pharmacological and non-pharmacological challenge tests, a review. J Psychiatr Res 2006, 40:550-567.

10. Yehuda R, Boisoneau D, Lowy MT, Giller EL Jr: Dose-response changes in plasma cortisol and lymphocyte glucocorticoid receptors following dexamethasone administration in combat veterans with and without posttraumatic stress disorder. Arch Gen Psychiatry 1995. 52:583-593.

11. van Zuiden M, Geuze E, Willemen HL, Vermetten E, Maas M, Heijnen CJ, Kavelaars A: Pre-existing high glucocorticoid receptor number predicting development of posttraumatic stress symptoms after military deployment. Am J Psychiatry 2011, 168:89-96.

12. van Zuiden M, Kavelaars A, Geuze E, Olff M, Heijnen CJ: Predicting PTSD: Pre-existing vulnerabilities in glucocorticoid-signaling and implications for preventive interventions. Brain Behav Immun 2013, 30:12-21.

13. Khan S, Liberzon I: Topiramate attenuates exaggerated acoustic startle in an animal model of PTSD. Psychopharmacology (Berl) 2004, 172:225-229.

14. Kohda K, Harada K, Kato K, Hoshino A, Motohashi J, Yamaji T, Morinobu S, Matsuoka N, Kato N: Glucocorticoid receptor activation is involved in producing abnormal phenotypes of single-prolonged stress rats: a putative post-traumatic stress disorder model. Neuroscience 2007, 148:22-33.

15. Liberzon I, Krstov M, Young EA: Stress-restress: effects on ACTH and fast feedback. Psychoneuroendocrinology 1997, 22:443-453.

16. Liberzon I, Lopez JF, Flagel SB, Vazquez DM, Young EA: Differential regulation of hippocampal glucocorticoid receptors $m R N A$ and fast feedback: relevance to post-traumatic stress disorder. J Neuroendocrinol 1999, 11:11-17.

17. Kozlovsky N, Matar MA, Kaplan Z, Zohar J, Cohen H: A distinct pattern of intracellular glucocorticoid-related responses is associated with extreme behavioral response to stress in an animal model of post-traumatic stress disorder. Eur Neuropsychopharmacol 2009, 19:759-771.

18. Wang HT, Han F, Shi YX: Activity of the 5-HT1A receptor is involved in the alteration of glucocorticoid receptor in hippocampus and corticotropinreleasing factor in hypothalamus in SPS rats. Int J Mol Med 2009, 24:227-231.

19. Knox D, Nault T, Henderson C, Liberzon I: Glucocorticoid receptors and extinction retention deficits in the single prolonged stress model. Neuroscience 2012, 223:163-173.

20. Adamec R, Muir C, Grimes M, Pearcey K: Involvement of noradrenergic and corticoid receptors in the consolidation of the lasting anxiogenic effects of predator stress. Behav Brain Res 2007, 179:192-207.

21. Liberzon I, Garfinkel SN: Functional neuroimaging in post-traumatic stress disorder. In Post-Traumatic Stress Disorder. Edited by LeDoux JE, Keane T, Shiromani P. New York: Humana Press; 2009:297-317.

22. Pitman RK, Rasmusson AM, Koenen KC, Shin LM, Orr SP, Gilbertson MW Milad MR, Liberzon I: Biological studies of post-traumatic stress disorder. Nat Rev Neurosci 2012, 13:769-787.

23. Levine $\mathrm{S}$ : Infantile experience and resistance to physiological stress. Science 1957, 126:405.

24. Levine S, Alpert M, Lewis GW: Infantile experience and the maturation of the pituitary adrenal axis. Science 1957, 126:1347.

25. Levine S: Maternal and environmental influences on the adrenocortical response to stress in weanling rats. Science 1967, 156:258-260.

26. Zarrow MX, Campbell PS, Denenberg VH: Handling in infancy: increased levels of the hypothalamic corticotropin releasing factor (CRF) following exposure to a novel situation. Proc Soc Exp Biol Med 1972, 141:356-358.

27. Meaney MJ, Diorio J, Francis D, Widdowson J, LaPlante P, Caldji C, Sharma S, Seckl JR, Plotsky PM: Early environmental regulation of forebrain glucocorticoid receptor gene expression: implications for adrenocortical responses to stress. Dev Neurosci 1996, 18:49-72.

28. Barnett SA, Burn J: Early stimulation and maternal behaviour. Nature 1967, 213:150-152.

29. Lehmann J, Feldon J: Long-term biobehavioral effects of maternal separation in the rat: consistent or confusing? Rev Neurosci 2000, 11:383-408.

30. Francis DD, Meaney MJ: Maternal care and the development of stress responses. Curr Opin Neurobiol 1999, 9:128-134.

31. Lee MHS, Williams DI: Changes in licking behaviour of rat mother following handling of young. Anim Behav 1974, 22:679-681. 
32. Meaney MJ, Aitken DH, Bodnoff SR, Iny LJ, Tatarewicz JE, Sapolsky RM: Early postnatal handling alters glucocorticoid receptor concentrations in selected brain regions. Behav Neurosci 1985, 99:765-770.

33. Papaioannou A, Gerozissis K, Prokopiou A, Bolaris S, Stylianopoulou F: Sex differences in the effects of neonatal handling on the animal's response to stress and the vulnerability for depressive behaviour. Behav Brain Res 2002, 129:131-139.

34. Panagiotaropoulos T, Papaioannou A, Pondiki S, Prokopiou A Stylianopoulou F, Gerozissis K: Effect of neonatal handling and sex on basal and chronic stress-induced corticosterone and leptin secretion. Neuroendocrinology 2004, 79:109-118.

35. Oines E, Murison R, Mrdalj J, Gronli J, Milde AM: Neonatal maternal separation in male rats increases intestinal permeability and affects behavior after chronic social stress. Physiol Behav 2012, 105:1058-1066.

36. Meaney MJ, Aitken DH, Viau V, Sharma S, Sarrieau A: Neonatal handling alters adrenocortical negative feedback sensitivity and hippocampal type II glucocorticoid receptor binding in the rat. Neuroendocrinology 1989, 50:597-604

37. Knox D, George SA, Fitzpatrick CJ, Rabinak CA, Maren S, Liberzon I: Single prolonged stress disrupts retention of extinguished fear in rats. Learn Mem 2012, 19:43-49.

38. Paxinos G, Watson C: The Rat Brain in Stereotaxic Coordinates. 2nd edition. San Diego: Academic Press; 1986.

39. Spencer RL, Kalman BA, Cotter CS, Deak T: Discrimination between changes in glucocorticoid receptor expression and activation in rat brain using western blot analysis. Brain Res 2000, 868:275-286.

40. Knox D, Nault T, Henderson C, Liberzon I: Glucocorticoid receptors and extinction retention deficits in the single prolonged stress model. Neuroscience 2012, 223:163-173.

41. Lehmann ML, Herkenham M: Environmental enrichment confers stress resiliency to social defeat through an infralimbic cortex-dependent neuroanatomical pathway. J Neurosci 2011, 31:6159-6173.

42. Binder EB: The role of FKBP5, a co-chaperone of the glucocorticoid receptor in the pathogenesis and therapy of affective and anxiety disorders. Psychoneuroendocrinology 2009, 34(Suppl 1):S186-S195.

doi:10.1186/2045-5380-3-22

Cite this article as: George et al:: Early handling attenuates enhancement of glucocorticoid receptors in the prefrontal cortex in an animal model of post-traumatic stress disorder. Biology of Mood \& Anxiety Disorders 2013 3:22.

\section{Submit your next manuscript to BioMed Central and take full advantage of:}

- Convenient online submission

- Thorough peer review

- No space constraints or color figure charges

- Immediate publication on acceptance

- Inclusion in PubMed, CAS, Scopus and Google Scholar

- Research which is freely available for redistribution 